\title{
How safe is elective induction - analysis in a tertiary care hospital in rural Punjab
}

\author{
Sharma $\mathbf{S}^{1}$
}

\section{Abstract \\ Objectives: The aims of the study were to analyse whether electively induced labour place the mother or her fetus at an increased risk as compared to patients in spontaneous labour and also to quantify the risk of caesarean section in the induced group.}

Methods: A prospective analysis comparing 150 electively induced parturients with 150 matched controls who laboured spontaneously in Gian Sagar Medical College and Hospital from July 2010 to July 2011 was done. The patients were between 37 and 41 weeks of pregnancy and had no complications necessitating induction of labour.

Results: Induction per se was not associated with a statistically significant increase in caesarean rates. Only when associated with nulliparity, low bishop score, and birth weight $>3.5 \mathrm{~kg}$, the risk of caesarean increases.

Elective induction is relatively safe and does not pose an increased risk of caesarean section in a carefully selected population. However when associated with risk factors the rate of caesarean section increases.

Key words: elective induction, caesarean section rates

\section{Introduction}

Elective induction of labour is defined as initiation of term labor without a medical or an obstetric indication. The rationale of elective induction is patients as well as obstetricians convenience. However the opinion is divided regarding elective induction ${ }^{1-3}$. Proponentssay that induction avoids potential adverse outcomes with impending post term, IUD of unknown cause $^{1}$. Especially in rural population where they have problems of transport and coming at odd hours to the hospital is not feasible, elective induction is well accepted. More importantly it

\footnotetext{
${ }^{1}$ Associate Professor Gian Sagar Medical College and Hospital, Ramnagar, Banur, Patiala, Punjab, India.

Correspondence: S Sharma

E-mail: sushmitasharma28@gmail.com

Competing interests: None.
}

allows a day time delivery with a better perinatal care, better planning by the physician, patient and the families. In urban areas with more pregnant women being working mothers or more responsible outside home desires advance arrangements for work, travel and home hence elective induction is favoured. In India, more so in rural areas, adding to this are some social beliefs which makes some days inauspicious and desire a planned day for deliveries. Opponents believe that it is an unnatural process, and once the induction has started there is a tendency to more readily accept further interventions in the form of operative or assisted deliveries ${ }^{2-3}$. Aims of the study were to analyse whether electively induced labour place the mother or her fetus at an increased risk as compared to patients in spontaneous labour and to quantify the risk of caesarean section in the induced group.

\section{Methods}

The study population consists of 150 patients in induction (study) group and 150 patients in the spontaneous (control) group between 37 and 41 weeks of gestational age. The control case was selected by choosing the next case who laboured spontaneously. The inclusion criteria was impending post term pregnancy, social reasons, on demand inductions, clinically suspected decreased liquor but AFI $>5$ on USG, suspected macrosomia (but USG estimated weight of $<4 \mathrm{~kg}$ ), patients at term but complaining of decreased fetal movements however NST reactive. Exclusion criteria were non cephalic presentations, all high risk pregnancies demanding induction. Out of the 150 patients 139 were induced with prostaglandins and oxytocin and 11 were induced with ARM and oxytocin. Statistical analysis was done using $\times 2$ test, Mann-Whitney U test.

\section{Results}

The risk of caesarean section rates in nulliparous women who were induced was statistically significant as depicted in Table 1. Caesarean delivery rates were significantly higher in nulliparous women with induced labour with poor bishop score (Table 2). Among induction group, patients with Bishop score $<5$ were associated with statistically significant (29\%) risk of caesarean section rates when compared to those who had Bishop score $>5$ $(7.2 \%)$. However this significance was seen in nulliparous women only. (Table 3). In the present study birth weight of more than $3.5 \mathrm{~kg}$ was associated with statistically significant increase in caesarean section rates $\{(54.2 \% \quad(P=0.00003)\}$ (Table 4$)$. 
Table1. Baseline data

\begin{tabular}{llcc}
\hline Nullipara & Induced group (\%) & Spontaneous group (\%) & Significance \\
\hline No. of cases & 113 & 113 & $\mathrm{P}<0.001^{* *}$ \\
Cesarean section & $33(29.1 \%)$ & $16(14.8 \%)$ & $\mathrm{NS}$ \\
Instrumental delivery & $3(2.7 \%)$ & $1(1.1 \%)$ & 37 \\
Multipara & 37 & $2(5.3 \%)$ & NS \\
No. of cases & $4(10.6 \%)$ & 0 & NS \\
Cesarean section & $1(1.7 \%)$ & & \\
Instrumental delivery & & & \\
\hline
\end{tabular}

**Highly significant

Table 2. Comparasion of induced group according to Bishop score $(\mathrm{N}=150)$

\begin{tabular}{llll}
\hline Parity & $\begin{array}{c}\text { Bishop score }<5 \\
\mathrm{~N}=117\end{array}$ & $\begin{array}{c}\text { Bishop score }>5 \\
\mathrm{~N}=33\end{array}$ & $\begin{array}{c}\text { Significance } \\
\text { Nullipara }\end{array}$ \\
Multipara & $30 / 89(33.7 \%)$ & $2 / 24(8.3 \%)$ & $\mathrm{P}=0.016^{*}$ \\
Total & $3 / 28(10.8 \%)$ & 0 & $\mathrm{NS}$ \\
\hline
\end{tabular}

*significant

Table 3. Incidence of caesarean section in different weight categories

\begin{tabular}{lccc}
\hline Birth weight $(\mathrm{kg})$ & Number $(\mathrm{N})$ & LSCS (\%) & Vaginal delivery \\
\hline $2-2.5$ & 72 & $4(5.5)$ & 68 \\
$2.6-3.0$ & 114 & $21(18.4)$ & 93 \\
$3.1-3.5$ & 96 & $17(17.7)$ & 79 \\
$>3.5$ & 18 & $10(55.5)$ & 8 \\
Total & 300 & $52(17.3)$ & 248 \\
\hline
\end{tabular}

Table 4. Details of labour and delivery

\begin{tabular}{lccl}
\hline Parameters & Induced & Spontaneous & Significance \\
\hline First stage & & & \\
Nullipara & $8.6 \mathrm{hrs}$ & $7.2 \mathrm{hrs}$ & $\mathrm{P}<0.001^{* *}$ \\
Multipara & $7.6 \mathrm{hrs}$ & $4.9 \mathrm{hrs}$ & $\mathrm{P}<0.001^{* *}$ \\
Second stage & & & \\
Nullipara & $48 \mathrm{~min}$ & $33.0 \mathrm{~min}$ & $\mathrm{P}=0.013$ \\
Multipara & $23.3 \mathrm{~min}$ & $26.0 \mathrm{~min}$. & $\mathrm{NS}$ \\
\hline
\end{tabular}

${ }^{*}$ Highly significant 
There is a statistically significant increase in duration of both first stage and second stage of labour in nulliparous induced women as compared with her control $8.6 \mathrm{~h}$ versus $7.20 \mathrm{~h}$ $(\mathrm{P}<0.001)$. In multipara the duration of first stage was prolonged $7.6 \mathrm{~h}$ versus $4.9 \mathrm{~h}(\mathrm{P}<0.001)$.

Further both the groups were analysed in the nulliparous women by comparing the caesarean section rates in females with bishop $<5$ after excluding the birth weight $>3.5 \mathrm{~kg}$ and age $>30$ years. The caesarean rates in the induced group was not statistically higher $(P \neq 0.05)$ than the spontaneous group proving that induction per se is not associated with increased caesarean rates. Only when associated with other factors the risk increases (Table 5).

The most common indication for caesarean section in the induced group was fetal distress and in the spontaneous labour group it was meconium stained liquor. $52 \%$ of women in the induced group were delivered in the day time as compared to $32 \%$ in the spontaneous group women. Maternal and neonatal complications are given in Table 6.

Table 5. Indications for caesarean section

\begin{tabular}{lcc}
\hline Indication & Induced & Spontaneous \\
\hline Fetal distress & 13 & 3 \\
Arrest of dilatation & 7 & 1 \\
Arrest of descent & 3 & 3 \\
Muconium stained liquor & 7 & 10 \\
Failed induction & 6 & 0 \\
\hline
\end{tabular}

Table 6. Maternal and fetal outcome

\begin{tabular}{lll}
\hline Parameters & Induced group & Spontaneous group \\
\hline Fetal outcome & & \\
Birth weight & $2.8 \mathrm{~kg}$ & $2.7 \mathrm{~kg}$ \\
Apgar score $<7$ at 1min. & 8 & 7 \\
FHS abnormalities & 13 & 11 \\
Cord prolapse & 0 & 0 \\
Muconium stained liquor & 18 & 19 \\
NICU admissions & 6 & 5 (p=0.79 NS) \\
Maternal outcome & & 2 \\
Intrapartum fever & 3 & 4 \\
PPH & 2 & 0 \\
Shoulder dystocia & 1 & 4 \\
Perineal tear & 6 & 0 \\
Cervical tear & 1 & \\
\hline
\end{tabular}


second stage of labour in the induced group. This is similar to Vaharatian et $\mathrm{al}^{6}$ study but contradictory to Macer et $\mathrm{al}^{7}$ study who found no difference in both stages of labour. Prysak and Castranova et al had concluded that increased caesarean was because the population had significant risk factors (nulliparity, poor bishop score, birth weight $>3.5 \mathrm{~kg}$ ) for caesarean delivery that nullified the risk of elective induction itself. This is in conjunction with our study too. Women should be appro-priately counselled in order to make an informed choice between elective induction or waiting for spontaneous labor beyond term.

\section{Conclusion}

Elective induction does not appear to pose an increased risk to the mother or her fetus in a very carefully selected population. It is safe and convenient for the patient as well as for the physician. However, when associated with nulliparity, poor bishop score and estimated fetal weight of $>3.5 \mathrm{~kg}$, it has a statistically significant increase in caesarean section rate. Elective induction of labor can be considered in selected women after obtaining detailed informed consent.

\section{REFERENCES}

1. Caughey $A B$, Sundaram $V$, Kaimal $A J$, Gienger A, Cheng YW, et al. Systemic review: elective induction of labour versus expectant management of pregnancy. Ann Inter Med 2009; 151(4): 252-63.

2. Vrouenraets FPJM, Roumen FJME, Dehing CJG, et al. Bishop score and risk of caesarean delivery after induction of labour in nulliparous women. Obstet Gynecol. 2005; 105: 690-7.

3. Bodner-Adler B, Bodner K, Patiesky N, Kimberger O, Chalubinski K, et al. Influence of labor induction on obstetric outcomes in patients with prolonged pregnancy: a comparasion between elective labor induction and spontaneous onset of labor beyond term. Wlenklin Wochenschr 2005; 117(7): 287-92.

4. Gulmezoglu AM, Crowther CA, Middleton P, Heatley E. Induction of labour for improving birth outcomes for women at or beyond term. Cochrane Database Syst Rev. 2012. 13; 6CD004945.

5. Prysak M. Castronova FC. Elective induction versus spontaneous labor a case control analysis of safety and efficacy. Obstet Gynecol. 1998; 92: 47-52.

6. Vaharatian A, Zhang J, Tronendle JF, et al. labour progression and risk of caesarean delivery in electively induced nulliparas. Obstetgynaecol. 2005; 105: 698-704.

7. Macer JA, Macer CL, Chan LS. Elective induction versus spontaneous labour: a retrospective study of complications and outcome. Am J Obst Gynecol. 1992; 166: 1690-7. 